\title{
Genome-wide high-throughput screening of T cell epitopes
}

\section{T-scan allows discovery of epitopes that define T-cell-based immunity.}

$\mathrm{T}$ he complexities of the immune system are extremely fascinating. The body produces a variety of cells that perform surveillance, recognition and actual combat against infection and tumors. The same system is also responsible for autoimmunity, where it may start recognizing its own components as foreign. In order to fully understand how the immune system works and the origins of autoimmunity, it is important to understand what immune cells actually 'recognize'.

The immune system remembers what it has encountered in the past; and this memory is maintained through memory B and $\mathrm{T}$ lymphocytes. B cells carry antibodies as receptors on their surfaces; these antibodies also get secreted by cells and thus can easily be purified. Knowing the antigen being recognized by a given $\mathrm{B}$ or $\mathrm{T}$ cell can be challenging. High-throughput screening of B-cell specificities involves using a display system to present large candidate antigen libraries to purified antibodies, and are relatively straightforward to perform.

On the other hand, $\mathrm{T}$ cell receptors (TCRs) reside on the cell surface and require the use of whole cells to do any experimental studies. The binding affinities between $\mathrm{T}$ cells and their antigens are also relatively low. T cells recognize short peptides that are presented or displayed on cell surfaces through MHC molecules to distinguish self from non-self. For example, when a virus infects a cell, the cell displays peptides of viral origin on its surface, which get recognized as foreign by $\mathrm{T}$ cells and elicits a response. "Pathogens are there [in the body] for a while and then they are eliminated, so you don't necessarily know what the T cell recognized. They're just sort of recording of the history of what they've seen in the past. So, in order to figure out what the $\mathrm{T}$ cells are recognizing, you need to provide them with the antigens that they once saw", says Stephen Elledge, from Harvard University Medical School. Elledge and colleagues have recently developed T-scan, a high-throughput method for genome-wide identification of antigens recognized by $\mathrm{T}$ cells.

In T-scan, an expression library encodes 90 amino acid peptides covering the entire

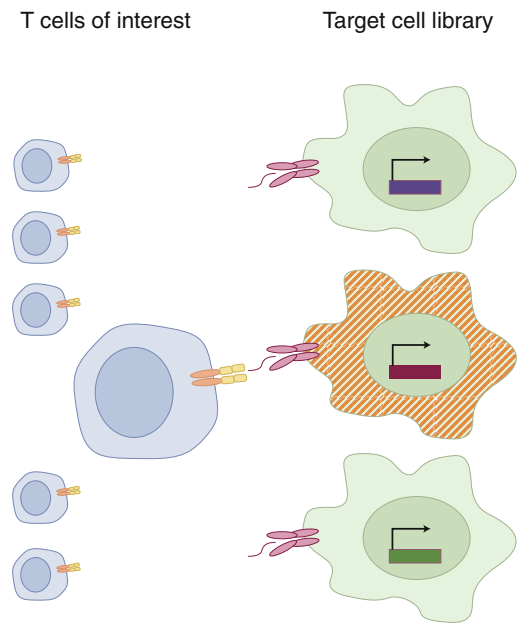

T cells of interest interact with a target cell library as part of T-scan. Figure adapted from Kula, T. et al. Cell 178, 1016-1028 (2019).

human or pathogenic proteome of interest. The peptides are delivered into target cells using a lentivirus-based transduction system, and each cell processes and displays a unique peptide on its MHC. Then, purified $\mathrm{T}$ cells from patients are tested for binding to this library. The binding is detected through the use of an enzyme called granzyme B, a serine protease that induces apoptosis in target cells. These reside in granules inside $\mathrm{T}$ cells, and following target recognition, they are released and inserted into the target cells to initiate apoptotic cell death. As the cells that bear the epitopes that the $\mathrm{T}$ cell recognizes begin to die, a fluorescent reporter gets activated following granzyme B cleavage. "When they fluoresce, we can FACS sort them, PCR amplify the peptidecoding DNA from the cells that are being killed, and then sequence them to figure out what the TCR is actually recognizing", explains Elledge. In this manner, they can precisely determine the epitopes that particular $\mathrm{T}$ cells recognize.

The researchers applied their method to three applications. They performed a screen for cytomegalovirus (CMV) antigens from bulk memory $\mathrm{T}$ cells in blood obtained directly from patients that had been previously infected with CMV. The CMV proteome-wide screen comprised of 5,000 peptides, and they found six epitopes, four of which were previously unknown. They also mapped the critical residues in the TCR-peptide interface for three CMV-specific TCRs, and were able to show that T-scan can detect subtle differences in TCR specificity for low-affinity interactions. Finally, they screened for the targets of a self-reactive TCR called MAGE, in a human proteome-wide screen of 250,000 peptides. They identified the known target for MAGE TCR as well as a related protein that differs by a single amino acid. Two other proteins that differ by three amino acids, not previously known to bind MAGE TCR, were discovered.

The method, however, is not without limitations, as Elledge points out, "It can't find all targets because the peptides we're making are 90 amino acids long. That's the size of our peptidome that tiles to the whole proteome. Plus, they are less likely to be modified, such as through phosphorylation, as they might be in the normal context of the protein". Therefore, the method is not perfect for certain modifications, but those are not easy to capture through other methods either.

T-scan is anticipated to help three predominant areas of research: infectious disease, autoimmunity and cancer immunology. Systematic profiling of T-cell targets and identification of auto-reactive TCRs, as well as screening for crossreactivity to viral and bacterial antigens, and profiling for neoantigens in a patientspecific experiments, will predictably allow tremendous advances in our understanding of disease progression as well as discovery of new therapeutic avenues.

Arunima Singh

Published online: 27 September 2019 https://doi.org/10.1038/s41592-019-0599-0

Research paper

Kula, T. et al. T-scan: A genome-wide method for the systematic discovery of T cell epitopes. Cell 178, 1016-1028 (2019). 